\title{
Measuring obesity in children: what standards to use?
}

\author{
Andrew Tomkins*
}

$\mathrm{C}_{\mathrm{n}}$ concern because of the rapidly increasing prevalence in many countries worldwide. ${ }^{1}$ Obesity contributes to early heart disease, the metabolic syndrome including hyperlipidemia and diabetes, hypertension and stroke. ${ }^{2}$ Policies and programs for improving the nutrition and health of children can only really be developed on an evidence base if there is some information on how many children are overweight and how much their health risk is increased by being at a specified level of overweight or frank obesity. Among adults this is relatively easy because of the wellestablished statistical risk of early morbidity and mortality in association with measurements of weight and height expressed as BMI $\left(\mathrm{kg} / \mathrm{m}^{2}\right)$ scores of $>25$ (overweight) or $>30$ (obese). ${ }^{3,4}$ There is clear evidence from longitudinal studies that childhood obesity is associated with increased risk of vascular and metabolic problems in adulthood. ${ }^{5}$ However, several methods are currently being used for measuring obesity in children; the challenge is to select the best one and keep to it for assessing and monitoring childhood obesity in clinical and public health practice.

There are several issues to face when deciding on what cutoffs give a "safe" level of body weight for stature in childhood. The first is age. In a comparison of six international data sets, median BMI is around 13 at birth and increases to 17 by age 1 , decreasing to 15.5 by age 6 , and then increasing to 21 at age 20.6 The second is ethnic diversity. There are differences in the distribution of body fat between subjects of different descent though how much this is due to dietary

\footnotetext{
* Professor of International Child Health, Centre for International Child Health, Institute of Child Health, University College London, London, UK.

Suggested citation: Tomkins A. Measuring obesity in children: what standards to use? J Pediatr (Rio J). 2006;82:246-8.

doi:10.2223/JPED.1516
}

differences rather than genetic predisposition is still disputed. The third is puberty. There are clear differences in body shape between boys and girls and the age at which puberty occurs changes the shape of any agespecific BMI curve.

A key question remains to be addressed at national and international levels - what standards should be used for diagnosing overweight among children? In May 2000, an important paper was published by Cole and colleagues describing weight and height at different ages in both boys and girls using population data from USA, Singapore, Netherlands, Hong Kong, UK and Brazil.6,7 The datasets used were large and rigorous, each survey containing over 10,000 subjects with ages ranging from 6-18 years. Percentile curves for BMI of these children were constructed using the LMS method. ${ }^{7}$ This summarizes the data in terms of three smooth age-specific curves called "L" (lambda), "M" ( $\mathrm{mu}$ ) and " $\mathrm{S}$ " (sigma). The $\mathrm{M}$ and $\mathrm{S}$ curves correspond to the median and coefficients of variation of the BMI at each 2-year age band. The $L$ curve allows for the substantial age-dependent skewness in the distribution of BMI. The mean values for the LMS calculation can then be provided in a table or a figure. The rationale for using this particular statistical approach is given in the paper by Cole et al. Not surprisingly there were differences in the curves using the median BMI by age for boys and girls between the six data sets. However, the basic shape of the curves was very similar.

Data were also expressed as the centile for overweight (using the adult BMI index of 25) and a centile for obesity (using the adult BMI of 30). These centile curves were much closer together than the median curves. Using a BMI of 25 as cutoff at 18 years, the percentage above the cutoff (overweight) ranged from $4.7 \%$ in Brazilian boys up to $18.1 \%$ among USA boys. The equivalent figures for girls were $15.2 \%$ and $16.5 \%$ respectively. Using the centile score for a BMI of 30 (obese) at 18 years, the percentage above cutoff point 
was $0.1 \%$ in Brazilian boys ranging up to $3.3 \%$ in USA boys. The equivalent figures in girls were $2.0 \%$ and $4.0 \%$ respectively.

The results of this analysis were supported by the International Obesity Task Force as it provided a table of international cutoff points for BMI for overweight and obesity by sex between 2 and 18 years. While this approach provides an age and sex-based cutoff for overweight or obesity which is linked to the adult risk, the authors pointed out that the degree of risk associated with these cutoffs in children, in terms of adverse health risk in adulthood, has not been validated in many countries and longitudinal studies are urgently needed. ${ }^{8}$

Subsequently, a large number of papers on childhood obesity have been published, several of them using different standards and choosing the more liberal cutoff points of the 85 th or 95 th centiles of national standards for overweight and obesity respectively. These have been critically compared with the BMI cutoffs. ${ }^{9}$ Jebb \& Prentice 10 also challenged the use of centiles rather than BMI-adjusted data on several grounds. Firstly they noted that different values are obtained according to whichever centile standards are used, thus making international comparisons impossible. Secondly, they pointed out the obvious self-fulfilling prophecy that if cutoff points using percentiles are used to measure overweight and obesity there will always be $15 \%$ overweight and $5 \%$ obesity. Thirdly they noted that the choice of 85 th and 95 th centiles, as used by several investigators, effectively increases the apparent number of overweight and obese children. They argued strongly for the use of the International Obesity Task Force's reference standard, as in Table 4 of the paper by Cole and colleagues. 6

The paper by Conde $\&$ Monteiro, ${ }^{11}$ from the University of São Paulo, published in this edition, is therefore of considerable value. They have taken data from over 25,000 children between 2-19 years, extracted from the National Nutrition and Health Survey dataset of 1989. They have also used the LMS method to calculate the BMI curve parameters and added some further analysis to compare these parameters at different ages. They use the BMI cutoff values of $17.5,25$, and 30 at 20 years. This provides a detailed dataset, tabulating the results at 3 monthly intervals for each sex. In this way, the Brazilian data have been compared against adult BMIs in which risk is known in a number of countries. The dataset they analyzed was particularly strong with very low rates of exclusions or rejection of data and the subdivision of the analysis into 3-month groupings means that subtle differences in nutritional status of populations can be compared very carefully. For instance, future analyses on the impact of changing age of menarche or obesity prevention programs will now be possible.
There are several distinctive advantages of using the LMS method for analyzing detailed datasets such as in the paper by Conde \& Monteiro, and the authors noted the ability to independently model the coefficient of variation better than the standard deviation. There are still those who argue for use of national datasets to be analyzed according to centiles alone rather than centiles which are related to adult BMI-based cutoffs. However, within any country where nutritional transition is occurring, methods limited to centile analysis alone are unlikely to detect real changes in the prevalence of obesity, and it is to be hoped that the paper by Conde \& Monteiro will be used for national planning and monitoring.

One particularly interesting aspect of the paper by Conde \& Monteiro is the use of the centiles using a BMI of 17.5 as a cutoff point for nutritional deficit. This is a particularly useful addition in communities where some children may be underweight, while others may be overweight. The Double Burden of Malnutrition is increasingly recognized internationally.

The dataset in the paper by Conde \& Monteiro now enables a series of studies to be established including longitudinal assessment of risk according to childhood BMI, and it is to be hoped that the data will enable follow-up studies to be established. ${ }^{12,13}$ These data will also enable an evaluation of the impact of secular change in child development including menarche on growth. More importantly, this paper now enables careful analysis of socioeconomic, dietary and physical activity studies to be established in Brazil and elsewhere comparing the progress and impact of public health interventions for children who are increasingly vulnerable because they are too fat.

\section{References}

1. Stettler N. Comment: the global epidemic of childhood obesity: is there a role for the paediatrician? Obes Rev. 2004;5 Suppl $1: 1-3$.

2. Berenson GS, Srinivasan SR, Wattigney WA, Harsha DW. Obesity and cardiovascular risk in children. Ann N Y Acad Sci. 1993;699: 93-103.

3. Garrow, JS. Energy balance and obesity in man. Amsterdam: Elsevier; 1978.

4. Garrow, JS. Obesity and related diseases. London: Churchill Livingstone; 1988.

5. Power C, Lake JK, Cole TJ. Measurement and long-term health risks of child and adolescent fatness. Int J Obes Relat Metab Disord. 1997;21:507-26.

6. Cole TJ, Bellizzi MC, Flegal KM, Dietz WH. Establishing a standard definition for child overweight and obesity worldwide: international survey. BMJ. 2000;320:1240-3. 
7. Cole TJ, Freeman JV, Preece MA. British 1990 growth reference centiles for weight, height, body mass index and head circumference fitted by maximum penalized likelihood. Stat Med. 1998; 17:407-29.

8. Must A, Jacques PF, Dallal GE, Bajema CJ, Dietz WH. Long-term morbidity and mortality of overweight adolescents. A follow-up of the Harvard Growth Study of 1922 to 1935. N Engl J Med. 1992;327:1350-5.

9. Wang $Y$, Wang JQ. A comparison of international references for the assessment of child and adolescent overweight and obesity in different populations. Eur J Clin Nutr. 2002;56:973-82.
10. Jebb SA, Prentice AM. Single definition of overweight and obesity should be used. BMJ. 2001;323:999.

11. Conde WL, Monteiro CA. Body mass index cutoff points for evaluation of nutritional status in Brazilian children and adolescents. J Pediatr (Rio J). 2006;82:266-72.

12. Bellizzi MC, Dietz WH. Workshop on childhood obesity: summary of the discussion. Am J Clin Nutr. 1999;70:173S-5S.

13. Reilly JJ. Assessment of childhood obesity: national reference data or international approach? Obes Res. 2002;10:838-40. 